\title{
V703 Sco: Metal abundances and space motions
}

\author{
E. Rodríguez \\ Instituto de Astrofísica de Andalucía, CSIC, \\ P.O. Box 3004, E-18080 Granada, Spain, E-mail:eloy@iaa.es
}

\begin{abstract}
A comprehensive study of the metal abundances and space motions on the highamplitude $\delta$ Sct star V703 Sco has been carried out. Our results suggest this star is a normal high-amplitude $\delta$ Sct-type variable of Population I with nearly solar abundances and small space motions located in the galactic plane. This is in very good agreement with that suggested by the period ratio $P_{0} / P_{1}=0.768$ which takes place for its two main periodicities.
\end{abstract}

Individual Objects: V793 Sco, SXPhe, CY Aqr

\section{Introduction}

V703 Sco is a two-mode high-amplitude $\delta$ Sct star $\left(\mathrm{V}=7 .^{m} 85, \Delta \mathrm{V}=0 .{ }^{m} 5\right.$, Rodríguez et al. 2000) discovered by Ponsen (1961). However, some controversy concerning its true nature comes up when its physical parameters are determined from near-IR spectroscopy (Amado 2007), suggesting this star as a metal-deficient SX Phe-type variable with $[\mathrm{Me} / \mathrm{H}] \sim-0.5$.

In order to discriminate between Population I and II for V703 Sco, an analysis on the metal abundances and space motions is crucial for this star. This way, we comprehensively studied these aspects on the basis of the information available in the bibliography.

\section{Metal abundances}

Due to the lack of spectroscopic studies concerning abundance analysis for V703 Sco, with the only exception of those carried out to obtain the Preston's (1959) $\Delta$ S-index, our analysis on metal abundances is mainly based on photometric studies. Table 1 lists the values found in the literature for parameters related to metal abundances in different photometric systems and from different sources, together with those for the $\Delta S$ parameter. In all cases, they all agree to consider V703 Sco as a normal high-amplitude $\delta$ Sct star of Population I rather than a Population II SX Phe-type pulsator. 
Table 1: Metal abundances for V703 Sco. Sources are:

1) Rodríguez et al.(1994) and López de Coca et al.(1990), based on Eggen(1978),

2) this work, 3) Rodríguez et al. (2000), based on Olsen (1983) and Olsen \& Perry (1984), 4) Jones (1973), 5) Bessell (1969), and 6) Lub (1977).

\begin{tabular}{lcc}
\hline Parameter & Value & Source \\
\hline$\delta \mathrm{m}_{1}$ & $0 .^{m} 011$ & 1 \\
$\beta$ & $2 .^{m} 736$ & 1 \\
{$[M e / H]($ as A star $)$} & $-0.04 \mathrm{dex}$ & 2 \\
{$[M e / H]$ (as F star) } & $-0.06 \mathrm{dex}$ & 2 \\
$\delta \mathrm{m}_{1}$ & $0 .^{m} 031$ & 3 \\
$\beta$ & $2 .^{m} 711$ & 3 \\
{$[M e / H]($ as A star $)$} & $-0.25 \mathrm{dex}$ & 2 \\
{$[M e / H]($ as F star $)$} & $-0.34 \mathrm{dex}$ & 2 \\
$(\mathrm{k}-\mathrm{b})_{2}$ & $0 .{ }^{m} 25$ & 4 \\
$\Delta \mathrm{S}$ & 0 & 5 \\
$\Delta \mathrm{S}$ & 1 & 6 \\
\hline
\end{tabular}

Concerning Strömgren photometry, the $\delta \mathrm{m}_{1}$ and $\beta$ indices are used to obtain the metal content $[\mathrm{Me} / \mathrm{H}]$ by considering $\mathrm{V} 703 \mathrm{Sco}$ as both an A or F-type star and using the calibrations of Smalley (1993) (for A stars, $\beta>2 .^{m} 72$ ) or Nissen (1988) (for F stars, $\beta<2 .{ }^{m} 72$ ), respectively. The results from different sources are slightly different, probably caused by photometric indices obtained at different phases of the pulsation cycle. In fact, the photometric indices from Olsen (1983) and Olsen \& Perry (1984) are based on only one measurement collected at an unknown pulsational phase. Nevertheless, the results are all consistent with solar or nearly solar metal abundances. This agrees well with the values available in the literature for the $\Delta \mathrm{S}$-index of Preston (1959) or $(k-b)_{2}$-index of Jones (1973).

Concerning five-color Walraven photometry, no $[\mathrm{Me} / \mathrm{H}]$ determinations are available in the bibliography, but it is very interesting to note that the behaviour of V703 Sco in the (B-L)-(V-B) plane (Ponsen 1963) is similar to other Population I high-amplitude $\delta$ Sct stars, as opposed to the behaviour shown by the metal deficient stars SX Phe or CY Aqr (Figure 5 of Bessell 1969). This study cannot be performed in Strömgren photometry because no $\mathrm{m}_{1}$-index curve is available for V703 Sco.

We can also analyse the possibility of solar metallicity for V703 Sco, but with abundance anomalies similar to the chemically peculiar $\lambda$ Boo-type stars. Nevertheless, this case is not probable because the $\lambda$ Boo stars pulsating as $\delta$ Sct-type variables all have very small amplitudes and short periods (unevolved stars) (Rodríguez \& Breger 2001, Paunzen et al. 2002). In fact, the star V703 Sco was unsuccessfully investigated in a series of works (Paunzen \& Gray 1997, Paunzen et al. 2001, Paunzen 2002) for $\lambda$ Boo characteristics. 
Table 2: Space motions for $\mathrm{V} 703 \mathrm{Sco} . \mathrm{V}_{r}=$ radial velocity; $\mathrm{V}_{t}=$ tangential velocity; $\mathrm{U}, \mathrm{V}, \mathrm{W}$ are the heliocentric space velocity components in a right-handed system, that is, with $\mathrm{U}$ towards the galactic center. Sources are:

1) Simbad 2007, 2) Van Herk (1965), 3) Hipparcos catalogue (Perryman et al. 1997),

4) Ponsen (1963), 5) Van Citters (1976), 6) this work, 7) Nördstrom et al. (2004),

8) McNamara (1985), 9) Rodríguez et al. (2000), and 10) Rodríguez \& Breger 2001.

\begin{tabular}{lcc}
\hline Parameter & Value & Source \\
\hline $\mathrm{l}$ & $356 .^{\circ} 6$ & 1 \\
$\mathrm{~b}$ & $-1 .^{\circ} 3$ & 1 \\
$\mu_{\alpha}$ & $26 \pm 21 \mathrm{mas} / \mathrm{yr}$ & 2 \\
$\mu_{\delta}$ & $11 \pm 21 \mathrm{mas} / \mathrm{yr}$ & 2 \\
$\mu_{\alpha}$ & $3.6 \pm 1.1 \mathrm{mas} / \mathrm{yr}$ & 3 \\
$\mu_{\delta}$ & $16.7 \pm 0.6 \mathrm{mas} / \mathrm{yr}$ & 3 \\
$\mathrm{~V}_{r}$ & $-35 \mathrm{~km} / \mathrm{s}$ & 4 \\
$\mathrm{~V}_{r}$ & $-53 \mathrm{~km} / \mathrm{s}$ & 5 \\
$<\mathrm{V}_{r}>$ & $-44 \mathrm{~km} / \mathrm{s}$ & 6 \\
$\mathrm{~V}_{r}$ & $-62 \mathrm{~km} / \mathrm{s}$ & 7 \\
vsini & $\leq 16 \mathrm{~km} / \mathrm{s}$ & 8 \\
vsini & $9 \mathrm{~km} / \mathrm{s}$ & 7 \\
$\pi$ & $3.91 \pm 0.98 \mathrm{mas}$ & 3 \\
$\mathrm{~V}$ & $7 . .^{m} 85$ & 9 \\
$\mathrm{M}_{v}$ & $0 . .^{m} 7 \pm 0.3$ & 10 \\
$\mathrm{~d}$ & $270 \mathrm{pc}$ & 6 \\
$\mathrm{~V}_{t}$ & $22 \mathrm{~km} / \mathrm{s}$ & 6 \\
$\mathrm{U}$ & $-43 \mathrm{~km} / \mathrm{s}$ & 6 \\
$\mathrm{~V}$ & $23 \mathrm{~km} / \mathrm{s}$ & 6 \\
$\mathrm{~W}$ & $9 \mathrm{~km} / \mathrm{s}$ & 6 \\
\hline
\end{tabular}

\section{Space motions}

Table 2 lists the parameters related with the space motion and galactic location of V703 Sco. For completeness, the values available for vsini are also listed. As expected for high-amplitude pulsators in the Lower Instability Strip, this star rotates very slowly. On the other hand, it is located just in the galactic plane with small space motions, which also agrees with the suggestion of V703 Sco being an object of Population I. In our determination for the U,V,W space velocity components, the proper motions obtained by the Hipparcos satellite (Perryman et al. 1997) were used. Concerning the radial velocity, the value of $\mathrm{V}_{r}=-62 \mathrm{~km} / \mathrm{s}$ listed in Nördstrom et al. (2004) has not been taken into account because this is based on only two measurements at unknown pulsational phases. Hence, this is probably affected by the intrinsic pulsational movement. However, the $\mathrm{V}_{r}$ values from Ponsen (1963) and 
Van Citters (1976) are based on mean values over a full pulsational cycle. Moreover, the absolute magnitude $\mathrm{M}_{v}(\pi)=0.8( \pm 0.5)$ derived from the Hipparcos parallax agrees well with the photometric one $\mathrm{M}_{v}(\mathrm{ph})=0 .{ }^{m} 7$, but with a larger error bar than the typical $\sigma_{M_{v}}=0 .{ }^{m} 3$ assumed for photometry.

\section{Conclusions}

To sum up the above analysis, V703 Sco seems to be a normal high-amplitude $\delta$ Sct star of Population I with nearly solar abundances and small space motions located in the galactic plane. This is consistent with its period ratio of $P_{0} / P_{1}=0.768$ obtained from the two main periodicities of this $\operatorname{star}\left(P_{0}=0 .{ }^{d} 1152\right.$ and $P_{1}=0 .{ }^{d} 1500$ as principal and secondary periods, respectively). They can be well fitted by solar-abundance evolution tracks assuming $\mathrm{P}_{0}$ and $\mathrm{P}_{1}$ as the first overtone and fundamental mode of radial pulsation (Cox et al. 1979, Andreasen 1983, Petersen \& Christensen-Dalsgaard 1996, Poretti et al. 2005).

Acknowledgments. This investigation was supported by the Junta de Andalucía and the Spanish Dirección General de Investigación (DGI) under project AYA200606375. This research has made use of both the Simbad database, operated at CDS, Strasbourg, France, and the Astrophysics Data System, provided by NASA, USA.

\section{References}

Andreasen, G. K. 1983, A\&A 121, 250

Amado, P. J. 2007, CoAst 151, 57

Bessell, M. S. 1969, ApJS 160, 195

Cox, A. N., King, D. S., \& Hodson, S. W. 1979, ApJ 228, 870

Eggen, O. J. 1978, IBVS 1517

Jones, D. H. O. 1973, ApJS 25, 487

López de Coca, P., Rolland, A., Rodríguez, E., \& Garrido, R. 1990, A\&AS 83, 51

Lub, J. 1977, Ph.D. Thesis Leiden Univ.

Nissen, P. E. 1988, A\&A 199, 146

McNamara, D. H. 1985, PASP 97, 715

Nordström, B., Mayor, M., Andersen, J., et al. 2004, A\&A 418, 989

Olsen, E. H. 1983, A\&AS 54, 55

Olsen, E. H., \& Perry, C. L. 1984, A\&AS 56, 229

Paunzen, E. 2002, A\&A 373, 633

Paunzen, E., \& Gray, R. O. 1997, A\&AS 126, 407

Paunzen, E., Duffee, B., Heiter, U., et al. 2001, A\&A 373, 625 
Paunzen, E., Handler, G., Weiss, W. W., et al. 2002, A\&A 392, 515

Petersen, J. O., \& Christensen-Dalsgaard, J. 1996, A\&A 312, 463

Perryman, M. A. C., Lindegren, L., Kovalevsky, J., et al. 1997, A\&A 323, L49

Ponsen, J. 1961, BAN 15, 325

Ponsen, J. 1963, BAN 17, 29

Poretti, E., Suárez, J. C., Niarchos, P. G., et al. 2005, A\&A 440, 1097

Preston, G. W. 1959, ApJ 130, 507

Rodríguez, E., \& Breger, M. 2001, A\&A 366, 178

Rodríguez, E., López de Coca, P., Rolland, A., et al. 1994, A\&AS 106, 21

Rodríguez, E., López-González, M. J., \& López de Coca, P. 2000, A\&AS 144, 469

Smalley, B. 1993, A\&A 274, 391

Van Citters, G. W. 1976, AJ 81, 766

Van Herk, G. 1965, BAN 18, 71 